\title{
Tecnura
}

\section{Estimación emisiones de compuestos orgánicos volátiles, plantel de distribución de combustibles Recope, el Alto de Ochomogo, Cartago, Costa Rica}

\section{Estimation of emissions of volatile organic compounds in the fuel marketing terminall Recope, Alto de Ochomogo, Cartago, Costa Rica}

\author{
Laura Vanessa Quesada Carvajal ${ }^{1}$, Macario Pino Gómez² \\ Rodolfo Elizondo Hernández ${ }^{3}$
}

Fecha de recepción: 1 de octubre de 2017

Fecha de aceptación: 28 de noviembre de 2017

Cómo citar: Quesada C., L.V., Pino G., M. y Elizondo H., R. (2018). Estimación emisiones de compuestos orgánicos volátiles, plantel de distribución de combustibles Recope, el Alto de Ochomogo, Cartago, Costa Rica. Revista Tecnura, 22(55), 25-33. https://doi.org/10.14483/22487638.12543

\section{Resumen}

Contexto: Este estudio presenta la estimación de las emisiones evaporativas generadas en el plantel de distribución de combustibles en el Alto de Ochomogo, Cartago, Costa Rica, y la selección del sistema de recuperación adecuado para reducir la emisión de estos gases a la atmósfera, disminuyendo así los efectos adversos provocados por estos compuestos en el ambiente y la salud de las poblaciones cercanas.

Método: Se estimó la tasa de emisiones fugitivas en el proceso de carga de vehículos cisternas, usando factores de emisión de pérdidas de carga, y en el almacenamiento de combustibles, a través de un software especializado. Posteriormente, se seleccionó el sistema de tratamiento adecuado, considerando la capacidad de flujo de la corriente gaseosa que cada tecnología puede tratar

Resultados: Se determinó que la generación de COV es mayor en el área de cargaderos que en los tanques de almacenamiento, ya que estas corresponden a un
$95 \%$ y $5 \%$, respectivamente. En consecuencia, la propuesta del sistema de tratamiento de vapores de gasolina se enfoca en la zona de carga de combustible, con la selección de la condensación criogénica como tratamiento de recuperación no destructivo.

Conclusiones: La estimación de tasa de emisiones fugitivas permitió tener una base para establecer una estrategia para la reducción de estas emisiones en pro de la salud de los trabajadores que están constantemente expuestos a ellas.

Para disminuir las emisiones directas a la atmósfera durante la carga de las cisternas, se deben realizar los cambios necesarios para adaptarlos a un sistema hermético que permita enviar los vapores de gasolina que se generan por la presencia de producto residual en las paredes de los camiones y por la turbulencia que se provoca durante la carga del nuevo producto, a la unidad de recuperación de vapores que se instale.

Palabras clave: compuestos orgánicos volátiles (COV), inventario de emisiones, plantel de distribución de combustible, factores de emisión, Recope.

1 Ingeniera química, especialista en Emisiones Atmosféricas con énfasis en Tratamiento de Gases, máster en Dirección Medioambiental. Docente del Tecnológico de Costa Rica. Cartago, Costa Rica. Contacto: laura.quesada@itcr.ac.cr

2 Ingeniero sanitario, especialista en Tratamiento de Residuos. Docente del Tecnológico de Costa Rica. Cartago, Costa Rica. Contacto: mpino@itcr.ac.cr

3 Ingeniero electromecánico, especialista en Manejo de Refrigerantes, máster en Administración de Ingeniería Electromecánica. Docente del Tecnológico de Costa Rica. Cartago, Costa Rica Contacto: relizondo@itcr.ac.cr 


\begin{abstract}
Context: This study presents the estimation of the evaporative emissions generated in the fuel distribution plant in El Alto de Ochomogo, Cartago, Costa Rica and the selection of the adequate recovery system to reduce the emission of these gases into the atmosphere, thus decreasing the adverse effects caused by these compounds in the environment and the health of nearby populations.

Method: The fugitive emission rate estimated in the tanker vehicle loading process, using load loss emission factors, and fuel storage, through specialized software. Subsequently, we proceeded to make the selection of the appropriate treatment system, considering the flow capacity of the gaseous current that each technology can treat.

Results: It was determined that the generation of VOC is greater in the loading area than in the storage tanks, since they correspond to $95 \%$ and $5 \%$
\end{abstract}

respectively. Due to this, the proposal of the vapor treatment system focuses on the fuel-loading zone, selecting the cryogenic condensation as non-destructive recovery treatment.

Conclusions: The estimation of the fugitive emission rate allowed to have a base to establish a strategy for the reduction of these emissions in favor of the health of the workers who are constantly exposed to them.

To reduce direct emissions to the atmosphere during the loading of tanks. Necessary changes must be made to adapt them to an airtight system. That allows sending gasoline vapors that generated by the presence of residual product on the walls of trucks and due to the turbulence that arises during the loading of the new product, to the vapor recovery unit.

Keywords: emission factors, emissions inventory, fuel distribution facilities, Recope, volatile organic compounds (VOC).

\section{INTRODUCCIÓN}

Entre las metas instauradas en el Plan Nacional de Energía 2008-2021 (Dirección Sectorial de Energía, 2008), se establece que Costa Rica disponga de un suministro energético confiable y en armonía con la naturaleza, promoviendo el desarrollo de la infraestructura necesaria y la constante investigación e innovación. Uno de los objetivos estratégicos es la modernización y el fortalecimiento integral del sector energético, con el fin que los procesos sean más eficientes y amigables con el ambiente. La implementación de mejoras tecnológicas y de control en los procesos de transformación de materias primas (Arango, Sierra y Silva, 2014) son acciones importantes que debe desarrollar la Refinadora Costarricense de Petróleo (Recope), como la instalación de sistemas de recuperación de vapores de combustibles, tanto en la flotilla de transporte de combustibles como en los planteles de distribución, así como la instalación de sistemas de carga por debajo, con el fin de aumentar la seguridad durante estas operaciones y, al mismo tiempo, disminuir la emisión de compuestos orgánicos volátiles (COV) a la atmósfera.

Los COV son hidrocarburos que contienen de 2 a 20 átomos de carbono por molécula y se clasifican en COV ligeros, aquellos que poseen de 2 a 11 átomos de carbono, y pesados los que tienen de 12 a 20 átomos de carbono (Sánchez et al., 2004). A 293,15 K, presentan una presión de vapor de 0,01 kPa o más, o una volatilidad equivalente en las condiciones particulares de uso (Real Decreto 117/2003, 2003).

Dichos compuestos pueden dar lugar a otros secundarios, como el ozono troposférico que es un contaminante que afecta las vías respiratorias (Cárdenas, 2009)

Actualmente la empresa se dedica solo al almacenamiento y distribución de productos terminados livianos y pesados. El primer tipo se caracteriza por su baja viscosidad, menor de cuatro centistokes, estos corresponden a las gasolinas superior y regular, diésel automotriz, kerosene, AV-gas, jet 
fuel y gas licuado de petróleo (GLP). El producto negro presenta una viscosidad mayor a cuatro centistokes, los productos que se encuentran en esta categoría son el búnker (fuel oil), diésel térmico, gasóleo, asfalto y emulsiones asfálticas. Los combustibles más livianos son transportados mediante el oleoducto desde la refinería hasta los demás planteles (Recope, 2011).

El Plantel El Alto almacena y vende producto limpio (excepto los combustibles de aviación y GPL) y producto negro. Se caracteriza por abastecer principalmente a la zona metropolitana de San José y por ser el único que cuenta con una planta de producción de asfalto y emulsión asfáltica.

El análisis preliminar del sistema de distribución del Plantel El Alto arroja que podemos tener diversas fuentes individuales, y es importante que todas estas fuentes sean contabilizadas en un sistema de inventario. En general, en las terminales de distribución de productos derivados del petróleo, las emisiones principales tienen tres orígenes diferentes: fugas y derrames de la red de tuberías y depósitos; pérdidas por evaporación en los tanques de almacenamiento; vapores procedentes de las estaciones de carga y trasiego (Cordero, 2013).

Sin embargo, basados en un estudio realizado en 2002 por el Instituto Mexicano del Petróleo para determinar la contribución del ciclo de distribución de gasolina en la emisión de compuestos orgánicos volátiles en el área metropolitana de Ciudad de México, en el cual por medio de factores de emisión de las actividades vinculadas al trasiego y almacenamiento de productos derivados del petróleo presentes en la metodología AP-42 de la EPA, indican que las pérdidas evaporativas en la carga de los camiones representan el 90,8 \% del total de las emisiones, en los tanques de almacenamiento alrededor de un 6,7 \% y en los accesorios de las tuberías un 2,5\% (Instituto Mexicano del Petróleo, 2002).

El objetivo propuesto en este estudio fue realizar el diagnóstico preliminar de la operación del plantel mediante la estimación de la tasa de emisiones fugitivas en el proceso de carga de vehículos cisternas y en el almacenamiento de combustibles a través de un inventario de emisiones de COV. Una vez obtenida la cantidad de vapores orgánicos que se emiten, se procedió a realizar la selección del sistema de tratamiento de dichas emisiones, revisando la capacidad de flujo de la corriente gaseosa que cada tecnología puede tratar, así como otras características de su diseño, de manera que la elegida esté en la capacidad de reducir la emisión de estos gases a la atmósfera y, así, disminuir los efectos adversos provocados por estos compuestos en el ambiente y la salud.

\section{METODOLOGÍA}

\section{Estimación de las emisiones de COV en el área de cargaderos de producto limpio}

Inicialmente se analizaron las hojas de especificaciones de cada producto que se maneja en el plantel, tomando como referencia el Manual de productos (Recope, 2011) para seleccionar aquellos que serían objeto de estudio, dada su volatilidad e inflamabilidad.

La estimación de las emisiones se realizó a partir de la metodología expuesta en el capítulo 5.2 del AP-42 de la EPA (US EPA, 1995a), la ecuación (1) se empleará para estimar los factores de emisión.

$$
E F U L=12,46 X \frac{S P M}{T}
$$

Donde:

$E F U L=$ factor de emisión de pérdida de carga (lb/1000 gal).

$S=$ factor de saturación, depende del tipo de llenado de los camiones cisterna.

$P=$ presión de vapor real de la gasolina (libras por pulgada cuadrada absoluta (psia).

$M=$ masa molar de los vapores (lb/lb-mol).

$T=$ temperatura promedio del líquido cargado $\left({ }^{\circ} \mathrm{R}\right)$.

La presión de vapor real de los productos se calculó mediante los métodos que se encuentran 
en la sección 7.1 del AP-42 (US EPA, 1995a), correspondiente a la estimación de las emisiones en los tanques de almacenamiento de líquidos orgánicos y a partir de la ecuación (2).

$$
P=e^{\left(A-\frac{B}{T_{l}}\right)}
$$

Donde:

$P=$ presión de vapor real (psi).

$A=$ constante en la ecuación (adimensional).
$B=$ constante en la ecuación $\left({ }^{\circ} \mathrm{R}\right)$.

$T_{1}=$ temperatura promedio de líquido $\left({ }^{\circ} \mathrm{R}\right)$.

Las constantes A y B de la ecuación (2) están en función de la presión de vapor de Reid y de la pendiente "S", la cual corresponde a la pendiente de destilación a un $10 \%$ de volumen evaporado del producto de interés y cuyo valor reportado para gasolina es 3,0 y para su determinar sus valores se utilizan las ecuaciones (3) y (4).

$$
\begin{gathered}
A=15,64-1.854 S^{0,5}-\left(0,8742-0,3280 S^{0,5}\right) \ln (P V R) \\
B=8,742-1.042 S^{0,5}-\left(1,049-179,4 S^{0,5}\right) \ln (P V R)
\end{gathered}
$$

Donde:

$S=3,0$.

$P V R=$ presión de vapor de Reid (psi).
Interpolando y usando los valores reportados en la tabla 1, se determinó el peso molecular de un producto determinado, utilizando la ecuación

\begin{tabular}{|c|c|c|c|c|c|c|c|c|}
\hline \multirow{2}{*}{$\begin{array}{c}\text { Presión de } \\
\text { vapor Reid de la } \\
\text { gasolina. PVR (psi) }\end{array}$} & \multirow{2}{*}{$\begin{array}{c}\text { Peso molecular } \\
\text { del vapor a } \\
60^{\circ} \mathrm{F}\end{array}$} & \multicolumn{7}{|c|}{ Presión de vapor real (psi) } \\
\hline & & $40^{\circ} \mathrm{F}$ & $50{ }^{\circ} \mathbf{F}$ & $60^{\circ} \mathrm{F}$ & $70^{\circ} \mathrm{F}$ & $80^{\circ} \mathrm{F}$ & $90^{\circ} \mathrm{F}$ & $100{ }^{\circ} \mathrm{F}$ \\
\hline 13 & 62 & 4,7 & 5,7 & 6,9 & 8,3 & 9,9 & 11,7 & 13,8 \\
\hline 10 & 66 & 3,4 & 4,2 & 5,2 & 6,2 & 7,4 & 8,8 & 10,5 \\
\hline 8,3 & 68 & 2,7888 & 3,444 & 4,2188 & 5,1284 & 6,1891 & 7,4184 & 8,8344 \\
\hline
\end{tabular}
(5) (Randian International LLC, 1997).

Tabla 1. Propiedades físicas de la gasolina

Fuente: US EPA (1995a).

$$
M M_{i}=M M_{X}+\left(\frac{P V R_{X}-P V R_{i}}{P V R_{X}-P V R_{n}}\right)\left(M M_{n}-M M_{X}\right)
$$

Donde:

$\mathrm{MM}_{\mathrm{i}}=$ peso molecular del vapor interpolado para PVR de interés.

$M M_{x}=$ peso molecular del vapor a la PVR máxima en el intervalo de interpolación.

$\mathrm{MM}_{\mathrm{n}}$ : = peso molecular del vapor a la PVR mínima en el intervalo de interpolación.

$\mathrm{PVP}_{\mathrm{i}}=$ PVR interpolada (9).
$\mathrm{PVP}_{\mathrm{X}}=\mathrm{PVR}$ valor superior.

$P V P_{n}=P V R$ valor inferior.

Una vez obtenidas las variables de la ecuación (1) se realizó el cálculo de los factores de emisión para cada uno de los productos de estudio, y al final, las pérdidas totales de la carga de camiones cisterna se calcularon a partir de la ecuación (6) (Randian International LLC, 1997).

Tasa de Emisión $=\sum_{i=1}^{n} E F_{U L, C L V} \times$ Combustible $_{i}$ 


\section{Donde:}

Tasa de emisión = emisiones totales de la carga de camiones cisterna (lb/año).

$E F_{U L, C L V}=$ factor de emisión de la carga controlada de vehículos cisterna para el modo de operación o el tipo de combustible i (lb/1000 gal).

Combustible $i=$ cantidad anual de combustible entregado por cada modo de operación o tipo de combustible i (gal/año).

Para obtener el volumen de gases por tratar, se divide la masa de emisiones por la densidad de los vapores de las gasolinas. Dicha densidad se calcula por medio de la ecuación (7), tal como lo indica el capítulo 7.1 del AP-42 de la EPA (US EPA, 1995a).

$$
\rho=\frac{P M \times P}{T \times R}
$$

Donde:

$P M=$ peso molecular de los vapores de gasolina (lb/lb-mol).

$T=$ temperatura promedio diaria de la gasolina $\left({ }^{\circ} \mathrm{R}\right)$.

$P=$ presión de vapor de la gasolina a la temperatura promedio diaria (psi).

$R=$ constante de los gases ideales 10,731 psi p3 /(lb/ lb-mol $\left.{ }^{\circ} \mathrm{R}\right)$.

\section{Estimación de las emisiones en los tanques de almacenamiento de gasolina regular y superior}

Se utilizó el software TANKS 4.0.9d de la EPA (US EPA, 1996), para modelar las pérdidas de vapores de gasolina en los tanques de almacenamiento. Inicialmente se seleccionó el tipo de tanque a analizar, indicando tanques de techo flotante interior; posteriormente, el programa despliega una ventana con cinco pestañas, las cuales se describen así:

- Identificación: Se introdujo el número de identificación del tanque, una descripción general, la provincia, la ciudad y la compañía a la cual pertenece.
- Características físicas: Se incorporaron datos de diseño del tanque considerando el tipo de tanque previamente seleccionado.

- Tipo de sellos: Se seleccionó el sistema de sellado que se utiliza para controlar las pérdidas por evaporación entre el espacio anular entre el perímetro o borde de la cubierta y el cuerpo del tanque, para permitir que se mueva el techo flotante dentro de este.

- Características de la plataforma: Se incorporan datos de tipo de techo flotante y de la configuración de los accesorios.

- Selección del sitio: Se indicó que la estimación de las emisiones se realizara en el plantel de Recope ubicado en el Alto de Ochomogo y se introdujeron los datos climatológicos correspondientes al lugar del estudio provisto por el Instituto Meteorológico Nacional.

- Contenido del tanque: Se seleccionó un destilado de petróleo que corresponde a la gasolina PVR 9.

- Cálculos mensuales: En esta última sección, se generó el reporte I de la estimación de las emisiones. Se debe indicar al programa que los resultados se quieren presentar mensualmente y de manera detallada.

\section{Selección del sistema de tratamiento de emisiones de COV para el Plantel El Alto}

Para la selección del sistema de tratamiento de emisiones de COV se caracterizaron las tecnologías existentes que no fuesen destructivas, se determinó su rango de aplicación según caudal, concentración y las características generales de diseño de las mismas. Además, se analizaron las ventajas y desventajas de cada sistema de tratamiento de esos gases.

Se comparó el valor del flujo volumétrico de la corriente de vapores de gasolina por tratar, calculado con el inventario de emisiones en la zona de cargaderos con los valores reportados por la literatura para seleccionar la tecnología de tratamiento aplicable para nuestro caso de estudio. 


\section{RESULTADOS}

\section{Estimación de las emisiones de COV}

Para obtener los factores de emisión calculados a partir de la ecuación (1) se utilizaron las propiedades fisicoquímicas de los combustibles seleccionados reportados en la tabla 2 .

Para la estimación de las emisiones de COV se tomó un año base de venta de producto limpio, por medio del Departamento de Distribución del
Plantel El Alto, se obtuvo el historial de ventas de cada producto en estudio. La tasa de emisión generada por la carga de los camiones cisternas y el flujo volumétrico estimado a tratar se presenta en la tabla 3.

Como se indicó en la metodología, cuando se introdujeron los datos solicitados por el programa TANKS 4.0.9d de la EPA (US EPA, 1996), se obtuvo la estimación de las emisiones de COV en los tanques de almacenamiento, cuyos valores se muestran en la tabla 4. Las primeras cuatro filas

Tabla 2. Propiedades de las gasolinas regular y superior empleadas en la estimación de las emisiones de COV

\begin{tabular}{lcccc}
\hline Producto & Temperatura $\left({ }^{\circ} \mathbf{R}\right)$ & $\begin{array}{c}\text { Presión de vapor de } \\
\text { Reid (psi) }\end{array}$ & $\begin{array}{c}\text { Masa molar } \\
(\mathbf{l b} / \mathbf{l b}-\mathbf{m o l})\end{array}$ & $\begin{array}{c}\text { Presión de valor real } \\
(\mathbf{p s i})\end{array}$ \\
\hline Regular & 529,47 & 9,42 & 66,69 & 5,83 \\
\hline Superior & 529,47 & 9,21 & 66,92 & 5,70 \\
\hline
\end{tabular}

Fuente: Cordero (2013).

Tabla 3. Estimación de las emisiones de vapores de gasolinas generadas en los patios de carga de combustible del Plantel El Alto

\begin{tabular}{lccc}
\hline \multicolumn{1}{c}{ Producto } & & Gasolina regular & Gasolinasúper \\
\hline Factor de emisión & $(\mathrm{lb} / 1000 \mathrm{gal})$ & 5,49 & 5,39 \\
\hline Volumen anual de ventas & $(\mathrm{gal})$ & 67873506 & 45824590 \\
\hline Emisiones & (kg/año) & 169031 & 112003 \\
\hline Total & (kg/año) & & 281034 \\
\hline
\end{tabular}

Fuente: elaboración propia.

Tabla 4. Estimación de las emisiones de COV de los tanques de almacenamiento de gasolina regular y superior del Plantel El Alto

\begin{tabular}{|c|c|c|c|c|c|c|c|c|c|c|c|c|c|}
\hline \multirow{2}{*}{ Tanque } & \multicolumn{13}{|c|}{ Pérdidas mensuales estimadas (kg) } \\
\hline & Enero & Febrero & o Marzo & Abril & Mayo & Junio & Julio & Agosto & Setiembre & Octubre & Noviembre & Diciembre & Total \\
\hline $101-S$ & 133 & 133 & 135 & 138 & 139 & 139 & 138 & 139 & 139 & 138 & 135 & 133 & 1638 \\
\hline $102-S$ & 133 & 133 & 135 & 138 & 139 & 139 & 138 & 139 & 139 & 138 & 135 & 133 & 1638 \\
\hline $103-S$ & 133 & 134 & 136 & 138 & 140 & 140 & 138 & 139 & 139 & 138 & 135 & 134 & 1644 \\
\hline $108-S$ & 135 & 135 & 138 & 140 & 142 & 142 & 141 & 141 & 142 & 140 & 137 & 136 & 1669 \\
\hline $106-R$ & 110 & 111 & 112 & 114 & 116 & 116 & 115 & 115 & 115 & 114 & 112 & 111 & 1362 \\
\hline 107-R & 137 & 137 & 139 & 142 & 144 & 144 & 142 & 143 & 143 & 142 & 139 & 137 & 1689 \\
\hline $121-R$ & 271 & 272 & 276 & 281 & 285 & 284 & 282 & 284 & 284 & 282 & 276 & 273 & 3350 \\
\hline \multirow[t]{2}{*}{$122-\mathrm{R}$} & 271 & 271 & 275 & 281 & 284 & 283 & 281 & 283 & 283 & 281 & 275 & 272 & 3339 \\
\hline & & & & & & & & & & \multicolumn{3}{|c|}{ Pérdida total/anual (kg) } & 16329 \\
\hline
\end{tabular}

Fuente: elaboración propia. 
muestran las pérdidas mensuales estimadas en cada tanque que contiene gasolina súperior (-S) y las filas restantes muestran los mismos resultados, pero en los tanques con gasolina regular (-R).

La comparación de los resultados de las tasas de emisión en la zona de cargaderos, y de los tanques de almacenamiento de gasolina regular y súper del Plantel El Alto, arrojó que el área en donde se generan mayores pérdidas de vapores de gasolina es la de los cargaderos, y corresponde a 281 ton/año, lo que representa un $95 \%$ de las emisiones. Esto debido a que, en la actualidad, se realiza la carga de combustibles por la parte superior de la cisterna y hace descender parcialmente la manguera de Ilenado para servir la gasolina dentro del tanque de carga. Durante esta operación, se presenta turbulencia y contacto entre el vapor y el líquido lo que resulta en altos niveles de generación y pérdida de COV (US EPA, 1999).

Por otro lado, el nivel de emisiones de vapores obtenido en los tanques de almacenamiento corresponde a 16,3 ton anuales representado un $5 \%$ del total de las emisiones fugitivas analizadas y es justificable, consideramos que estos poseen un techo flotante, que se coloca directamente sobre la superficie del líquido permitiendo que se desplace según las variaciones del nivel del líquido, siendo una barrera que disminuye el escape de vapores de gasolina producto de la operación de llenado y descarga de los tanques (US EPA, 1995b).

Es importante señalar que existen diversos métodos para estimar la tasa de emisión de los vapores de COV, que varían según su grado de confiabilidad y costo, siendo el monitoreo continuo de las emisiones generadas ideal desde el punto de vista de confiabilidad, pero incorporando los mayores costos de implementación. Sin embargo, el uso de factores de emisión nos permite realizar una primera estimación de los valores esperados (Carmona et al., 2016) y es justificable, si se considera el control continuo de las calidades de las gasolinas y los procedimientos establecidos por la empresa durante el desarrollo de la operación de carga de producto limpio en el Plantel El Alto.

\section{Selección del sistema de tratamiento}

Para la selección adecuada del sistema de tratamiento de emisiones de COV, se tomó el valor estimado de flujo de volumétrico de vapores de gasolina que se genera durante el proceso de carga de camiones cisterna, que corresponde a $54 \mathrm{~m}^{3} / \mathrm{h}$ y se ubicó dentro de los rangos aplicables de flujo para cada tecnología existente de acuerdo con los parámetros establecidos en la tabla 5.

Se optó por la criocondensación como la tecnología de recuperación de vapores de gasolina para el Plantel El Alto, ya que de acuerdo con el análisis realizado por Cordero (2013), el nitrógeno empleado como refrigerante es muy eficiente y no es una sustancia agotadora de la capa de ozono a diferencia de los refrigerantes comúnmente utilizados en los sistemas de condensación convencionales. Además, es una tecnología compacta, posee

Tabla 5. Características generales de los sistemas de tratamiento de emisiones de COV

\begin{tabular}{|c|c|c|c|c|}
\hline Sistema de tratamiento & Eficiencia promedio & $\begin{array}{c}\text { Rango de aplicación } \\
\left(\mathrm{m}^{3} / \mathrm{h}\right)\end{array}$ & $\begin{array}{c}\text { Concentración (g/ } \\
\mathbf{m}^{3)}\end{array}$ & $\begin{array}{c}\text { Presión } \\
\text { (atm) }\end{array}$ \\
\hline $\begin{array}{l}\text { Separación con membranas } \\
\text { selectivas }\end{array}$ & $90-99,9 \%$ & $10-100$ & No reportado & No reportado \\
\hline Condensación & $50-98 \%$ & $100-100000$ & $50-100$ & No reportado \\
\hline Criocondensación & $95-99,95 \%$ & $10-10000$ & $2-50$ & $0,02-20$ \\
\hline Adsorción regenerativa & $95-99,99 \%$ & $100-100000$ & $0,1-10$ & $1-20$ \\
\hline Absorción & $95-99,99 \%$ & $100-100000$ & $10-50$ & No reportado \\
\hline
\end{tabular}

Fuente: Jiménez (2007). 
una alta eficiencia para la recuperación de COV y permite reciclar el nitrógeno gaseoso. También, es importante considerar algunas atenciones durante la implementación de este sistema de recuperación; por ejemplo, se requiere de etapas de tratamiento previas para eliminar la humedad que puede estar presente en la corriente de gases a recuperar para evitar la formación de hielo dentro del intercambiador, y es necesario contar con un proveedor de nitrógeno líquido.

\section{CONCLUSIONES}

Las estimaciones realizadas y la caracterización de los diferentes combustibles permiten concluir que las gasolinas regular y súper son los productos que presentan mayor volatilidad, debido a que se reportan valores de PVR cercanos a 9 psi.

La generación de vapores orgánicos volátiles en el Plantel El Alto, es mayor en el área de cargaderos que en los tanques de almacenamiento, ya que las emisiones corresponden a un $95 \%$ y un $5 \%$ del total, respectivamente.

La masa de emisión de COV durante la carga de las gasolinas regular y súper fue de 281 ton/año. Mientras que la emisión anual total en los tanques de almacenamiento fue de 16,3 ton/año. Estos hallazgos son coherentes con los resultados planteados por Evequoz et al., 2005); Stiver y Mackay (1984), y el Instituto Mexicano del Petróleo (2002).

Para disminuir las emisiones directas a la atmósfera durante la carga de los tanques cisterna, se deben realizar los cambios necesarios en las cisternas para adaptarlos al sistema de carga por debajo, ya que este es hermético y permite enviar los vapores de gasolina que se generan por la presencia de producto residual en las paredes de los camiones y por la turbulencia que se provoca durante la carga del nuevo producto, a la unidad de recuperación de vapores que se instale.

Se seleccionó la criocondensación como la tecnología de tratamiento de vapores de gasolina más apropiada para el Plantel El Alto, ya que es una tecnología compacta, posee una alta eficiencia para la recuperación de COV y permite reciclar el nitrógeno gaseoso.

Se recomienda realizar mediciones directas con equipos especializados en la zona de cargaderos para determinar un flujo volumétrico de la corriente gaseosa por tratar y concentración de COV que sea más representativo en el momento que se dimensione el sistema de recuperación de vapores.

\section{FINANCIAMIENTO}

Esta investigación es parte del proyecto de investigación y desarrollo denominado "Diseño de un sistema de control de pérdidas de COV para reducir el impacto ambiental de las emisiones fugitivas en los patios de trasiego del Plantel de Recope Ochomogo", financiado por la Refinería Costarricense de Petróleo (Recope) y el Instituto Tecnológico de Costa Rica (TEC). Los autores agradecen a todas las personas u organizaciones que colaboraron de alguna manera para el desarrollo de este proyecto.

\section{REFERENCIAS}

Arango G., J., Sierra V., F.E. y Silva L., V. (2014). Análisis exploratorio de investigaciones sobre los motores de combustión interna que trabajan con biogás. Tecnura, 18(39), 152-164.

Cárdenas F., H. (2009). Consideraciones del material particulado en Bogotá. Alternativas tecnológicas de medición de calidad de la calidad del aire. Tecnura, 13(25), 107-118.

Carmona A., L., Rincón P., L., Castillo R., A., Galvis R., B., Sáenz P., H., Manrique F., R. y Pachón Q., J. (2016). Conciliación de inventarios top-down y bottom-up de emisiones de fuentes móviles en Bogotá, Colombia. Tecnura, 20(49), 59-74.

Cordero, L. (2013). Propuesta del sistema de tratamiento de emisiones de compuestos orgánicos volátiles (COV), en el plantel de Recope ubicado en El Alto de Ochomogo. Cartago: Instituto Tecnológico de Costa Rica. 
Dirección Sectorial de Energía (2008). Plan Nacional de Energía 2008-2021. San José: Sistema de Información Energética Nacional.

Evequoz, O., Sbarato, D., Koroch, A., Rivarola, E., Sbarato, V., Ortega, J., Salort, M. y Campos, M. (2005). Pérdidas evaporativas por almacenamiento y distribución de combustibles en estaciones de servicio. Análisis de su problemática y propuesta de marco regulatorio local. Córdoba: Centro de Información y Documentación Regional, Universidad Nacional de Córdoba.

Instituto Mexicano del Petróleo (2002). Contribution of the Gasoline Distribution Cycle to Volatile Organic Compound Emissions in the Metropolitan Area of Mexico City. Recuperado de http://www.tandfonline.con/doi/pdf/10.1080/10473289.2002.10470803

Jiménez, J. (2007). Análisis de la problemática de las emisiones de compuestos orgánicos volátiles en un centro de refino. Sevilla: Escuela Técnica Superior de Ingeniería.

Randian International LLC (1997). Cuaderno de trabajo para la capacitacion avanzada en la metodología para el proyecto del inventario de emisiones de México. México.

Real Decreto 117 de enero de 2003. Sobre limitación de emisiones de compuestos orgánicos volátiles debidas al uso de disolventes en determinadas actividades. Madrid. Recuperado de http://www.istas. net/risctox/abreenlace. asp? idenlace $=2926$
Refinadora Costarricense de Petróleo (Recope) (2007). Instructivo para la revisión de camiones cisterna que ingresan al plantel. AF-04-03-001. Cartago.

Refinadora Costarricense de Petróleo (Recope) (2011). Manual de productos. Recuperado de https://www. recope.go.cr/manual-de-productos/

SEMARNAT (1998). Inventario de Emisiones de la Zona Metropolitana del Valle de México. Recuperado de http://148.206.53.231/UAM7190.PDF

Stiver, W. y Mackay, D. (1984). Evaporation rate of spills of hydrocarbons and petroleum mistures. Environmental Science \& Technology, 18(11), 834-840.

United States Environmental Protection Agency (US EPA) (1995a). AP-42 Compilación de factores de emisión de contaminantes atmosféricos. Recuperado de https://www3.epa.gov/ttnchie1/ap42/

United States Environmental Protection Agency (US EPA) (1995b). Tanques de almacenamiento de líquidos orgánicos. Carolina del Norte.

United States Environmental Protection Agency (US EPA) (1996). Programa TANKS 3.0. Carolina del Norte: Oficina de Calidad del Aire.

United States Environmental Protection Agency (US EPA) (1999). Sección 3. Controles de COV. Carolina del Norte. Recuperado de http://www.epa.gov/ttn/ dir2/cs3-1ch1-s.pdf 